\title{
GENERALIZED LIGHT GRADIENT BOOST CLASSIFIER FOR TRAFFIC AWARE SEAMLESS MOBILITY MANAGEMENT IN HETEROGENEOUS NETWORK
}

\author{
D.Somashekhara Reddy ${ }^{1}$ \\ Department of Computer Applications, T.John College, Bangalore, Karnataka, INDIA \\ Somashekhara.reddy@gmail.com \\ Dr. Chandrasekhar ${ }^{2}$ \\ Professor, Dept. of Computer Science, Periyar University, Salem, Tamil Nadu, INDIA \\ ccsekar@gmail.com
}

\begin{abstract}
Seamless mobility management is an ability to provide the various services during the communication in wireless heterogeneous networks. Due to the random mobility of the mobile terminals, the connectivity between different mobile devices gets lost. In order to provide the lossless connectivity between the mobile devices, the handover from the point of current attachment to another point is necessary. To improve the Seamless mobility management and traffic control, an efficient model called Generalized Light Gradient Boost Decision Tree-based Traffic-Aware Seamless Mobility (GLGBDTTASM) model is introduced in the heterogeneous network. When a mobile node in the network moves out of its communication range, the signal strength of the nodes is calculated. Based on the signal strength estimation, the Generalized Light Gradient Boost Decision Tree classifier categorizes the mobile nodes into the weak and strong signal strength with the threshold value. The boosting algorithm initially constructs' weak learners i.e. binary decision tree to identify the weak signal strength of the mobile node. Then the ensemble classifier combines the results of weak learners and minimizes the generalization error. This helps to perform the handover only with the weak signal strength of the node resulting in minimizes the redundant handover. In addition, the weak signal strength of the mobile node from the current attachment point handover towards the nearest available attachment point to improve the continuous data delivery. Followed by, bandwidth availability is measured for reducing the packet loss due to thenetwork traffic resulting in improves the seamless data delivery between the nodes. The simulation is carried out to evaluate the performance of the GLGBDT-TASM model with two related approaches. The results show that the GLGBDT-TASM model effectively improved traffic-aware seamless mobility in a heterogeneous network with minimum delay and packet loss as well as a higher data delivery rate as compared to state-of-the-art methods.
\end{abstract}

Keywords: Heterogeneous network, seamless mobility management, generalized light gradient boost decision tree, signal strength, handover

\section{INTRODUCTION}

Heterogeneous wireless networks are an integration of two different networks. For enhancing the performance of data communication, connections are to be exchanged among the various networks using seamless handoff. Seamless mobility is the ability to change the current network point of attachment into others without losing its connectivity. A Seamless mobility management system is effectively used for wireless communication techniques with mobile terminals to continuous access of information at any time, different location, network, and device. The seamless mobility plays a significant role in a wireless network in terms of data communication with minimum delay. In addition, one of the significant challenges in seamless mobility management is to handle and control the traffic since it leads to packet loss in data communication. To address this issue in this paper, the Generalized Light Gradient Boost Decision Tree-based Traffic-Aware Seamless Mobility (GLGBDT-TASM) model is introduced for seamless mobility management and traffic control with the help of machine learning techniques. 
A Locator and Identity Split Protocol and Location Service Areas (LISP-LSAs) mobility management protocol was designed in [1]. The designed mobility scheme considerably minimizing the data delivery cost and packet loss but the performance of the data delivery rate was not improved. A new Optimized Vertical Handover $(\mathrm{OVH})$ framework was introduced in [2] to perform the handover process with minimum time. Though the framework minimizes the handover delay, the traffic-aware seamless communication was not performed to further minimize the packet loss.

A Multi-Armed Bandit Model-based Vertical Handoff Approach (MABA) was designed in [3] for improving the seamless communication. The designed approach failed to minimize the performance of packet loss in the seamless data transfer. A Cross-Layer assisted multipath protocol was developed in [4] to provide the mobile device's service connection. But the protocol increases the throughput but the transmission delay was not minimized. A proactive caching mechanism based mobility supporting model was introduced in [5]. The mechanism considers the real-time network traffic but the performance of the packet loss remained unaddressed.

An Invasive Weed Optimization (IWO) algorithm was designed in [6] to perform the seamless handoff with probable attachment points for a particular mobile node. But the traffic-aware seamless handoff decision making was not performed. A novel vertical handover prediction method was developed in [7] to minimize the number of redundant handovers. The performance of the successful delivery due to the vertical handover was not improved.

A two mobile router network mobility method was developed in [8] for minimizing the transmission delay as well as packet loss. But the data delivery rate from one mobile to another node was not improved. A Flowbased and operator-centric mobility management model was introduced in [9] to improve the communication with less signaling overhead. The designed model was not used for the dynamic mobility management system. The integration of fuzzy logic and Markov Decision Process (MDP) was introduced in [10] for providing an accurate decision of handover to perform the data communication. The designed approach minimizes the mobility as well as traffic, but the delay was not minimized.

To solve the above-said problems in the existing literature, an efficient machine learning algorithm is introduced for a heterogeneous environment. The major contributions of this paper are described in the below subsection.

\subsection{Contribution of the work}

A GLGBDT-TASM model is developed for improving the seamless data communication. Comparison with other related works that our proposed model exhibits improved performance. The major contributions are summarized as follows.

- To reduce the packet loss rate with help of GLGBDT-TASM model. In this model, the performance of seamless data transmission is improved by identifying signal status using a generalized light gradient boost decision tree classification. During the data transmission, maximum bandwidth availability is selected between the nodes. In this way, seamless data transmission reduces the packet loss rate.

- To minimize the delay by means of proposed GLGBDT-TASM method. The handover process is used to seamlessly change points of the current network into the nearest available attachment point with minimum delay.

- To increase the packet delivery rate by using the proposed GLGBDT-TASM model. In this method, the node performs handovers to the other nearest point through the Euclidian distance measure with minimum time for continuous data transmission.

1.2

\section{Organization of the paper}

The rest of the paper is organized into six different sections. The reviews of the related works are presented in Section 2. In Section 3, the proposed GLGBDT-TASM model is described with a neat diagram. In section 4, simulation setup and parameter settings are presented. Simulation results are discussed in section 5 with different performance metrics. The conclusion of the paper is presented in Section 6. 


\section{RELATED WORKS}

A Multiple Attribute Decision Making (MADM) approach was introduced in [11] for selecting the network and vertical handover. The approach failed to perform the handover in the heterogeneous network. A Seamless Mobility Management Based on Session Initiation Protocol was designed in [12] to provide different services without a connectivity loss. Though the designed protocol minimizes the handover packet loss, the data delivery rate was not improved.

An adaptive handover prediction (AHP) method was developed in [13] for seamless mobility based wireless networks to improve the accuracy of handover decisions. The designed method failed to consider the traffic for minimizing the packet loss. A novel handover mechanism based on MIH/PMIPv6 was developed in [14] to perform the vertical handover for seamless mobility. Though the mechanism minimizes the packet loss, the transmission delay was not reduced.

A Context-Aware Network Selection was performed in [15] for dynamic decision-making of a mobile device to perform the communication between the devices. Though the model considers the network traffic, the performance of the packet loss was not minimized. A Multiple Attribute Decision-Making algorithm was introduced in [16] to perform the handover. The designed algorithm balances network load to avoid network congestion but the traffic-aware Decision Making was not performed.

QoE-driven channel allocation and spectrum handoff management framework was developed in [17] for seamless multimedia services. But it failed to use machine learning algorithms to minimize the redundant handoff. A new local anchor-based architecture was developed in [18] for performing the seamless handover with minimum cost. However, when the traffic was large, then the seamless mobility management was not effectively performed. Mobility management solutions were performed in [19] for minimizing the handoff latency and packet loss. But the bandwidth availability between the nodes was not considered. The vertical handoff decision-making technique was introduced in [20] based on the coverage area and the speed of the mobile nodes. Though the designed technique reduces the redundant handoff, the transmission delay was not minimized.

The Consumer surplus value (CSV)-based pricing scheme and prediction system was implemented in [21] for non-real time mobile-based services aiming to maximize user's satisfaction. However, in this method delay was not considered. In [22], Media Independent Handover (MIH) using a scheduling algorithm was introduced for achieving effective seamless data transmission. But, the packet delivery rate was not sufficient. A geometric model for the analysis of hand over failures based on the fading was presented in [23]. This model does not consider the mobility management and traffic. A horizontal handoff scheme was designed in [24] for providing efficient seamless handoff. This method used to reduce the handoff latency significantly. However, traffic aware data communication does not considered.

The major issues are overcome by introducing a novel GLGBDT-TASM model. The detailed processes of the GLGBDT-TASM model are explained in the following section.

\section{METHODOLOGY}

Seamless mobility management reduces the packet loss when the movements of mobile terminals from one attachment point to another. Mobility management enables the networks to locate a mobile attachment point for delivering the data packets without a connectivity loss. Due to seamless mobility, a mobile node has to handover the current conversation to the optimal network among the various heterogeneous networks for continuous data transmission. However, traffic control is one of the major concerns during the seamless mobility since it leads to packet loss during the transmission. Based on this motivation, an efficient technique called GLGBDT-TASM model is introduced. The main objective is to improve continuous data delivery and minimize the delay.

\subsection{System model}

The network model of proposed GLGBDT-TASM model is described in this section with number of distributed mobile nodes $M N_{1}, M N_{2}, M N_{3}, \ldots . M N_{n}$ in the square area $n * n$. The mobile nodes in the networks are connected to the attachment points $P_{1}, P_{2}, P_{3, \ldots} P_{n}$ to perform the seamless data $\mathrm{d}_{1}, \mathrm{~d}_{2}, \mathrm{~d}_{3, \ldots}, \ldots \mathrm{d}_{\mathrm{n}}$ transmission in a certain communication range $\mathrm{R}_{\mathrm{c}}$. Based on the above system model, the proposed GLGBDT-TASM model is designed. Every node in the heterogeneous network is free to move in various directions and changes its connections when the node moves beyond the communication range. Therefore, seamless management is essential to transmit the data. The block diagram of the GLGBDT-TASM model is shown in figure 1 


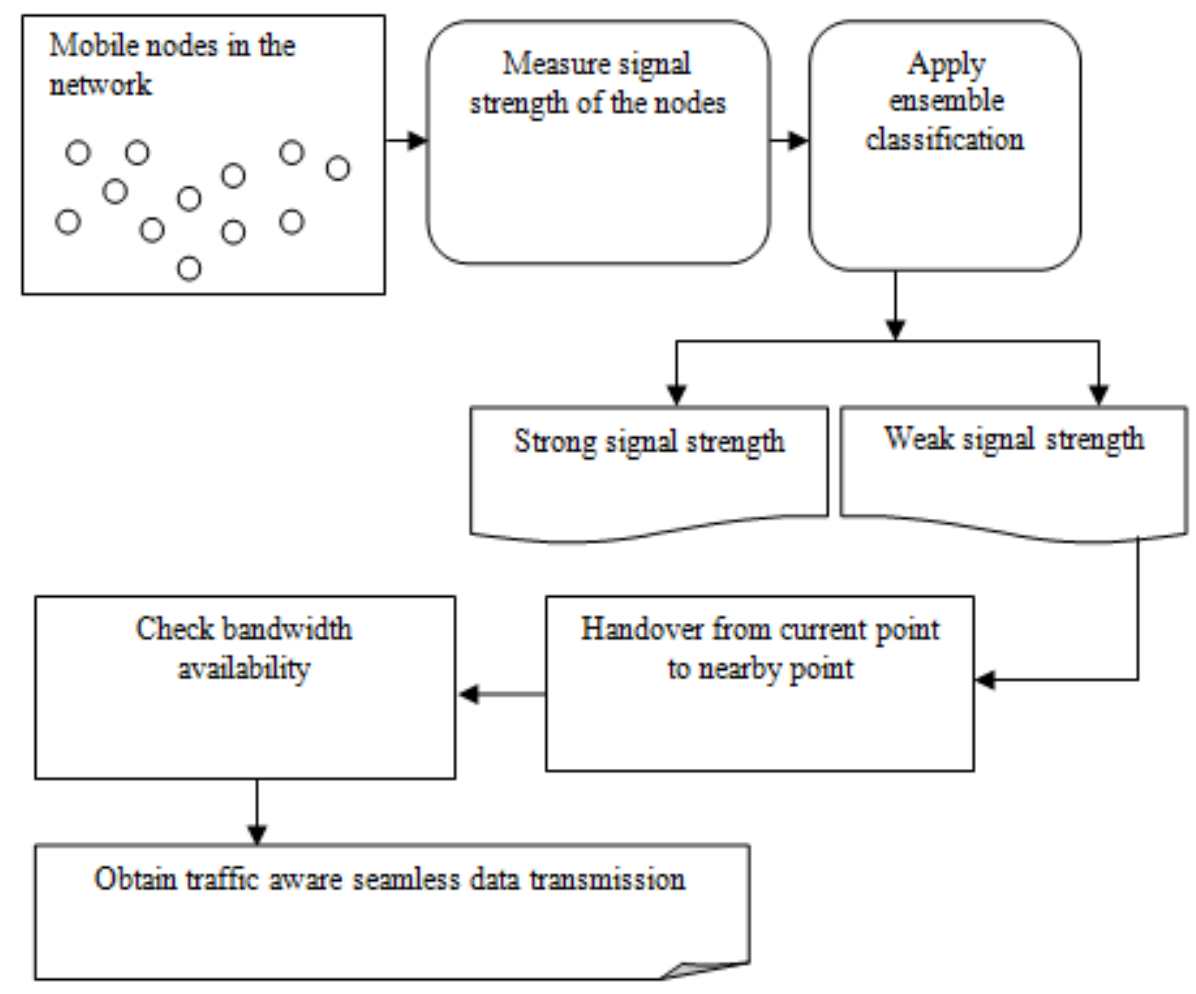

Figure 1 block diagram of the proposed GLGBDT-TASM model

Figure 1 illustrates a block diagram of the proposed GLGBDT-TASM model to achieve seamless data transmission between the mobile nodes in the heterogeneous network. The numbers of mobile nodes or devices are deployed in a heterogeneous network. For each node, the signal strength of the nodes is measured for seamless data transmission. Then, the ensemble classifier is used to classify strong signal strength or weak signal strength based on the threshold value. For the accurate classification results, weak learners are combined into a strong one. Generalization error is calculated by applying weight to the weak learners. Based on the error value, weak learner classifies the nodes as weak or strong signal strength. If the weak learner correctly classifies node status, then performs the handover process by measuring the Euclidean distance between the node and attachment points. During the continuous data transmission, maximum bandwidth availability is considered for minimizing the packet loss. In this way, the proposed GLGBDT-TASM model achieves efficient seamless data transmission with minimum delay. The detailed process of proposed GLGBDT-TASM method is expressed as follows,

The number of mobile nodes $M N_{1}, M N_{2}, M N_{3}, \ldots . M N_{n}$ are deployed in the network. The signal strength of the mobile node is mathematically calculated as follows,

$$
S_{r}=10 \log \left(\frac{P_{r}}{P_{t}}\right)
$$

In (1) $S_{r}$ denotes signal strength of the node, $P_{r}$ denotes a measured power and $P_{t}$ denotes reference power. The signal strength is measured in the unit of the decibel (dB). After measuring the signal strength, the nodes are classified using an ensemble classification technique 


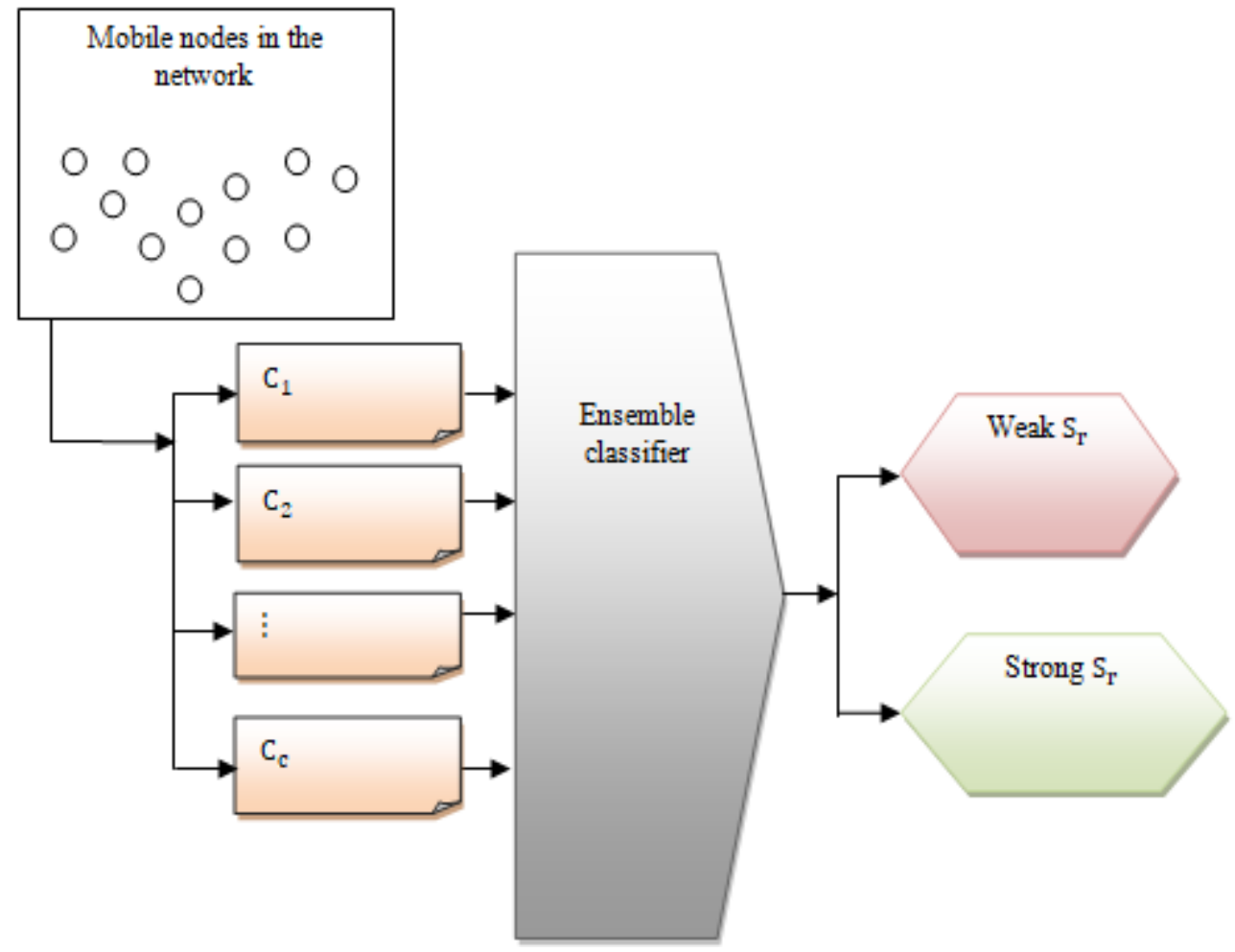

Figure 2 structure of the ensemble classification

The generalized light gradient boost decision tree classification is applied to classify the node as strong signal strength or weak signal strength. Boosting is a machine learning technique that converts the weak classification results into a strong one. The weak (i.e. base) classifier did not provide the true classification of the mobile nodes with their signal strength. On the contrary, a strong learner is also a classifier that arbitrarily provides accurate classification results. Therefore, the proposed GLGBDT-TASM model uses the ensemble classification algorithm for identifying the signal status of the mobile nodes based on the signal strength. The generalized light gradient boost decision tree classification uses the binary decision tree as a weak learner. The structure of the ensemble algorithm is shown in figure 2.

Figure 2 illustrates an ensemble classification of the nodes for performing the seamless data transmission with minimum delay. The ensemble algorithm considers the training sets $\left\{x_{i}, y_{i}\right\}$ where $x_{i}$ denotes an input i.e. number of mobile nodes and $y_{i}$ represents the final strong classification results. The ensemble classifier initially creates an empty set of ' $c$ ' weak learners $\mathrm{C}_{1}, \mathrm{C}_{2}, \mathrm{C}_{3}, \ldots . \mathrm{C}_{\mathrm{n}}$ with a number of mobile nodes. The binary decision tree used as a weak classifier is to classify the nodes into two classes' namely weak signal strength and strong signal strength hence the name is called as binary decision tree. The binary decision tree is constructed with three types of nodes such as root node, branch node, and leaf node. The root node makes a decision based on their signal strength with the threshold and the branch node provides the outcome of the test, and finally the leaf node displays the output class labels. The path from the root node to the leaf node denotes a certain classification rule. The root node verifies the signal strength with the threshold.

$$
C_{i}(x)=\left\{\begin{array}{l}
S_{r}>\delta_{t} ; \quad \text { strong signal strengt } \\
S_{r}<\delta_{t} ; \quad \text { weak signal strengt }
\end{array}\right.
$$

In (2), $C_{i}(x)$ represents the output of the weak classifier, $\delta_{t}$ denotes a threshold, $S_{r}$ is the signal strength of the node. The base classifier output has some errors in the classification results. In order to improve the classification accuracy and minimize the error, the output of the weak classification results are combined and to make a strong one. The output of the weak classifiers is summed as follows,

$$
y_{i}=\sum_{i=1}^{c} C_{i}(x)
$$

From (3), $y_{i}$ represents the output of the strong classifier, $C_{i}(x)$ is the output of the weak learners. The ensemble technique initially assigns the weight value to all the results of the weak classifier.

$$
y_{i}=\sum_{i=1}^{c} \alpha * C_{i}(x)
$$


In (4), $y_{i}$ represents the output of a strong classifier, $\alpha$ denotes a weight. Then the generalization error is calculated for each classification result. Generalization error is mathematically calculated based on the difference between the expected and empirical errors. Here the expected error is the actual error of the classifier and the empirical error is the predictable error. The generalization error is calculated as follows,

$$
E_{g n}=\left(E_{e t}-E_{e m}\right)
$$

In (5), $E_{g n}$ represents the generalization error, $E_{e t}$ denotes an expected error, $E_{e m}$ is the empirical error. After that, the weight value of each classifier is updated with respect to the generalization error. The initial weight is increased if the weak learner incorrectly classifies the nodes. On the contrary, the initial weight is decreased if the weak learner correctly classifies the nodes as weak or strong signal strength. Then the strong classifier finds the weak learner with minimum generalization error and the output of the strong classification results are expressed as follows,

$$
y_{i}=\sum_{i=1}^{c} \alpha_{t} * C_{i}(x)
$$

In (6) $\alpha_{t}$ represent the updated weight of the weak classifier. In this way, the ensemble classifier correctly identifies the node status and performing the handover process. After that, the weak signal strength of the mobile nodes is switched over from the current attachment point to the nearby attachment point. The nearby attachment point is identified through the Euclidean distance measure.

Let us consider the current coordinate for the mobile node is $\left(a_{1}, b_{1}\right)$ and the coordinate of available attachment point is $\left(a_{2}, b_{2}\right)$ in Euclidean space. Therefore, the distance between the node and the attachment point is computed as follows,

$$
D_{t}=\sqrt{\sum_{i=1}^{n}\left(a_{i}-b_{i}\right)^{2}}
$$

In (7), $D_{t}$ represents the distance. Then the technique finds the minimum distance for handover the node into the network.

$$
\arg \min \mathrm{D}_{\mathrm{t}}(8)
$$

In (8), arg min denotes an argument of the minimum function to find the minimum distance between the node and the currently available attachment point. After finding the nearby point, the weak signal strength of the mobile node is switched with minimum delay and performs the seamless data transmission between the two nodes. Therefore, the ensemble classification technique effectively finds the current signal status of the node for minimizing the redundant handover.

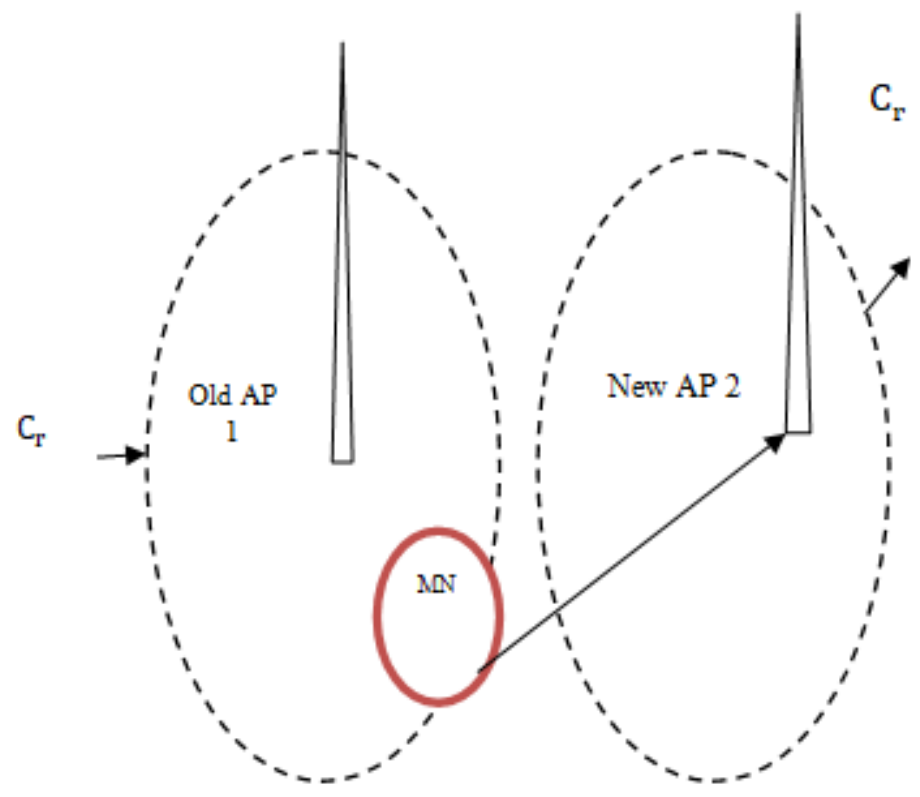

Figure 3 handover procedure

Figure 3 shows the mobile node $(\mathrm{MN})$ handover process where $\mathrm{C}_{\mathrm{r}}$ denotes a communication range. The weak signal strength of the mobile node handover from the old attachment point AP1 to new attachment point AP2 with minimum time and performing the continuous data transmission. While performing the data transmission, traffic occurs due to the utilization of the higher bandwidth which leads to higher data loss. In order to overcome the data packet loss, maximum bandwidth availability is selected between the nodes. The bandwidth availability is mathematically calculated using the following equation,

$$
b w_{a v l}=\left(b w_{t l}-b w_{u t}\right)
$$


In (9), $b w_{a v l}$ represents the bandwidth availability between the nodes, $b w_{t l}$ denotes a total bandwidth, $b w_{u t}$ is the utilized bandwidth. The maximum bandwidth availability between the nodes is selected for efficient seamless data transmission from one node to another with minimum packet loss as well as delay. In this way, the proposed technique effectively performs the seamless data transmission. The algorithmic process of the proposed technique described as follows,

Algorithm 1 Generalized Light Gradient Boost Decision Tree-based Traffic-Aware Seamless Mobility

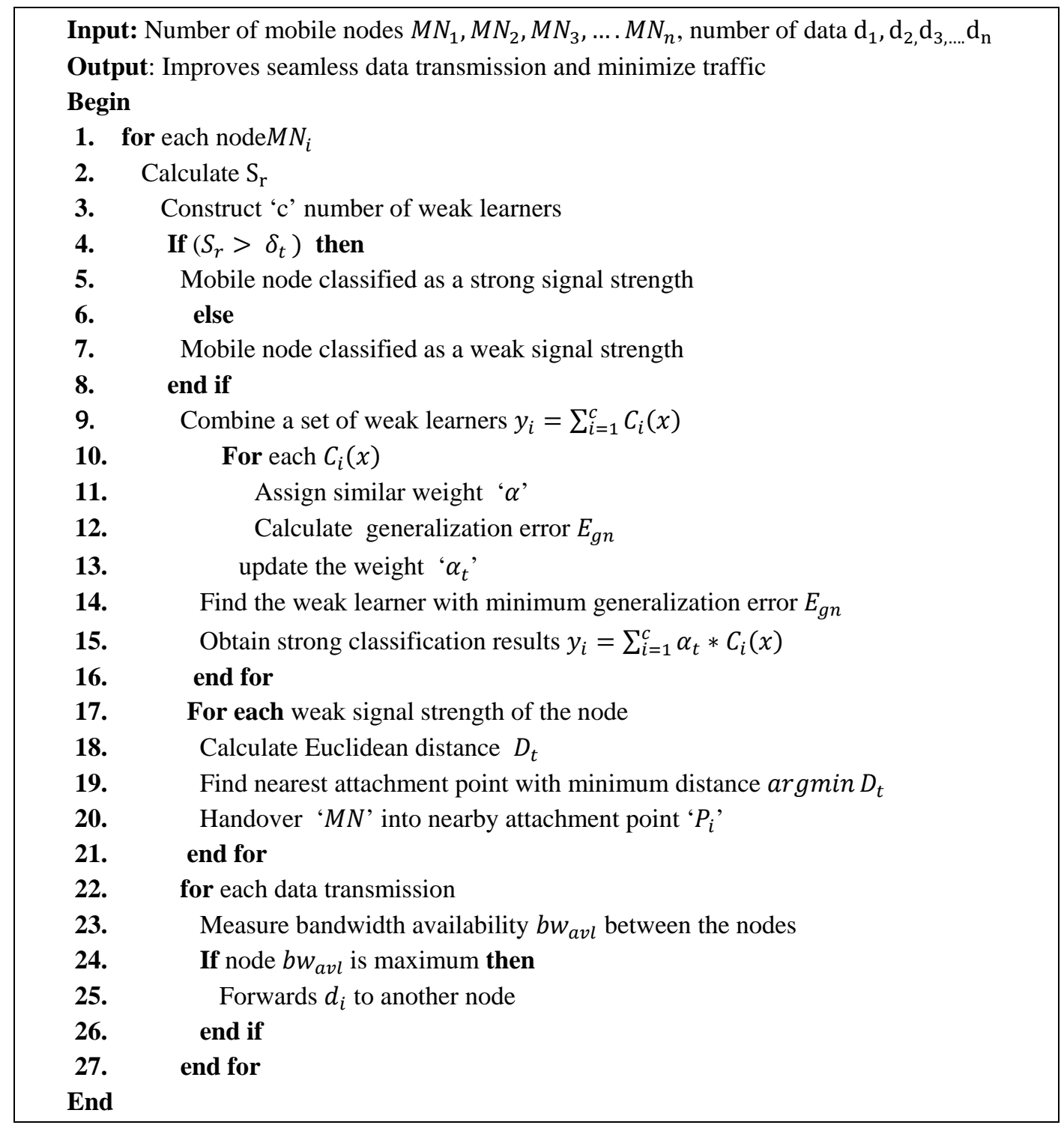

Algorithm 1 describes the generalized light gradient boost decision tree-based traffic-aware seamless mobility model to improve the traffic-aware data transmission with minimum packet loss. Initially, the number of mobile nodes signal strength is measured to find the status of the mobile node. Then the ensemble algorithm initially constructs an empty set of weak learners to correctly identify the weak and strong signal strength of the nodes. Followed by, the weak signal strength of the mobile nodes handovers to the new attachment point for seamless data transmission from one node to another. The Euclidean distance is measured between the mobile node and the available attachment points. While performing the continuous transmission, the bandwidth availability is measured to minimize the traffic. The nodes with maximum bandwidth availability are considered for minimizing the packet loss due to the traffic and improve the data transmission with minimum delay. 


\section{SIMULATION SETUP AND PARAMETER SETTINGS}

The proposed GLGBDT-TASM model is implemented in the Heterogeneous wireless networks for achieving efficient seamless data transmission with minimum delay. Totally 500 mobile nodes are deployed over the square area of $A^{2}(1100 m * 1100 \mathrm{~m})$. The Random Waypoint mobility model is used as a mobility model. The data packets are varied from 25 to 250 for performing the simulation. The simulation time is set as $100 \mathrm{sec}$. The DSR protocol is used to perform the seamless data transmission. The simulation is carried out using the proposed GLGBDT-TASM model and existing methods namely the LISP-LSA scheme [1] and the OVH framework [2] using NS2.34 simulator. The simulation parameters and their values are listed in table 1.

Table 1 Simulation Parameters

\begin{tabular}{ll}
\hline Simulation parameter & Value \\
\hline Simulator & NS2 .34 \\
\hline Network area & $1100 \mathrm{~m} * 1100 \mathrm{~m}$ \\
\hline Number of mobile nodes & $50,100,150,200,250,300,350,400,450,500$ \\
\hline Data packets & $25,50,75,100,125,150,175,200,225,250$ \\
\hline Protocol & DSR \\
\hline Simulation time & $100 \mathrm{sec}$ \\
\hline Mobility model & Random Way Point model \\
\hline Nodes speed & $0-20 \mathrm{~m} / \mathrm{s}$ \\
\hline Number of runs & 10 \\
\hline
\end{tabular}

The simulation results of the three different techniques GLGBDT-TASM model compared with LISP-LSA scheme [1] and OVH framework [2] are discussed in this section with different parameters such as

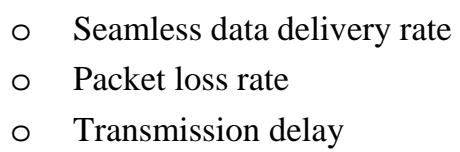

For each section, the mathematical calculation is given to show the performance of the proposed technique against the existing methods. The performance of the results is discussed in the following section.

\subsection{Impact of data delivery rate}

Data delivery rate referred to as a number of data are received to the total number of data sent from the node. The data delivery rate is mathematically computed using the following equation,

Data delivery rate $=\left(\frac{\text { Number of data packets received }}{n}\right) * 100$ (10)

In (10), $n$ denotes the number of packets sent. The Seamless data delivery rate is measured in the unit of percentage (\%).

\section{Sample calculation:}

Proposed GLGBDT-TASM: The number of data packets received is 22 , and the number of data packets sent is 25 . Then the data delivery rate is mathematically calculated as,

$$
\text { data deivery rate }=\frac{22}{25} * 100=88 \%
$$

Existing LISP-LSA scheme: The number of data packets received is 21, and the number of data packets sent is 25. Then the data delivery rate is mathematically calculated as,

$$
\text { data deivery rate }=\frac{21}{25} * 100=84 \%
$$

Existing OVH framework: The number of data packets received is 20, and a number of data packets sent is 25 . Then the data delivery rate is mathematically calculated as,

$$
\text { data deivery rate }=\frac{20}{25} * 100=80 \%
$$

The statistical results show that the data delivery rate of the three methods with the number of packets sent. Let us consider 25 data packets, 22 data packets are correctly received using the GLGBDT-TASM model and the delivery rate is $88 \%$. Similarly, 21 and 20 data packets are received using the LISP- LSA scheme [1] and OVH framework [2] and their percentage are $84 \%$ and $80 \%$ respectively. The performance of the various results of the data delivery rate is shown in figure 4. 


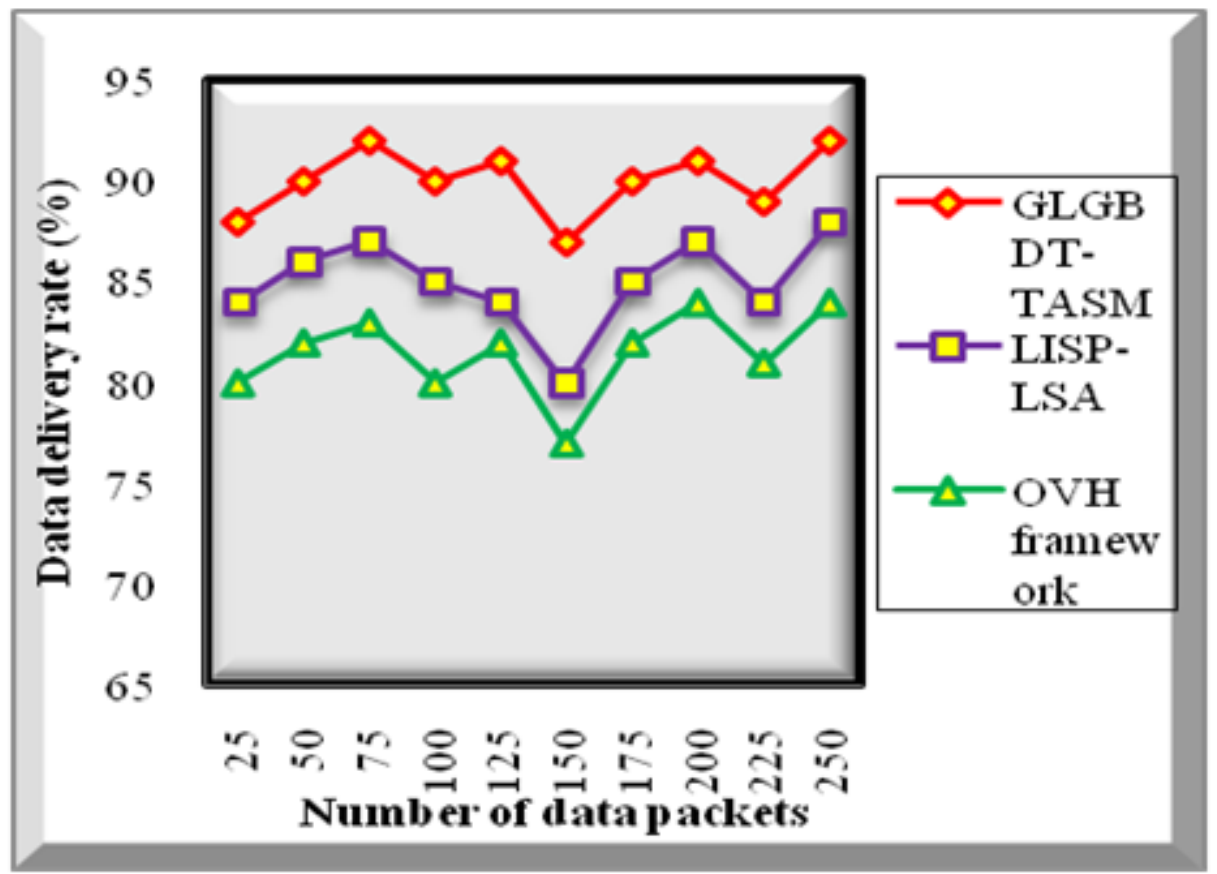

Figure 4 Simulation results of data delivery rate

Figure 4 depicts the simulation results of data delivery rate with respect to a number of data packets taken as input in the range from 25 to 250. As shown in the two-dimensional graph, the data delivery rate of the three different methods namely GLGBDT-TASM model, LISP-LSA [1] and OVH framework [2] are represented in three different colors such as red, violet and green respectively. The graphical results confirm that the GLGBDT-TASM model improves the data packet delivery rate as compared to other methods. This is because of the seamless data transmission is performed in GLGBDT-TASM model through the handover process. When the nodes move out of communication range, the data packets transmission is not successful since the mobile node has weak signal strength from the current attachment point. The GLGBDT-TASM model accurately finds the weak signal strength of the mobile nodes by performing the ensemble classification algorithm. Then the node performs handovers to the other nearest point through the Euclidian distance measure with minimum time for continuous data transmission. This process of the GLGBDT-TASM model enhances the continuous data transmission from one node to another.

Totally ten results of the data delivery rate are obtained with a various number of the data packet sent as shown in figure 4.The The data delivery rate of the proposed model is compared to the existing results. The comparison result proves that the

GLGBDT-TASM model improves the data delivery rate by $6 \%$ as compared to the LISP-LSA [1] and 10\% compared to the OVH framework [2].

\subsection{Impact of packet loss rate}

The packet loss rate is referred to as a number of data packet loss to the total number of the data packet sent from the node. The packet loss rate is mathematically computed using the following equation,

$$
P L R=\left(\frac{\text { Number of data packets lost }}{n}\right) * 100 \text { (11) }
$$

In (11),PLR denotes a packet loss rate, $n$ denotes the number of a packet sent. The packet loss rate is measured in the unit of percentage (\%).

Sample calculation:

Proposed GLGBDT-TASM: Number of data packets lost is 3, and the number of data packets sent is 25. Then the packet loss rate is calculated as,

$$
P L R=\frac{3}{25} * 100=12 \%
$$

Existing LISP-LSA scheme: The number of data packets lost is 4, and the number of data packets sent is 25 . Then the packet loss rate is calculated as,

$$
P L R=\frac{4}{25} * 100=16 \%
$$

Existing OVH framework: The number of data packets lost is 5, and the number of data packets sent is 25 . Then the packet loss rate is calculated as,

$$
P L R=\frac{5}{25} * 100=20 \%
$$




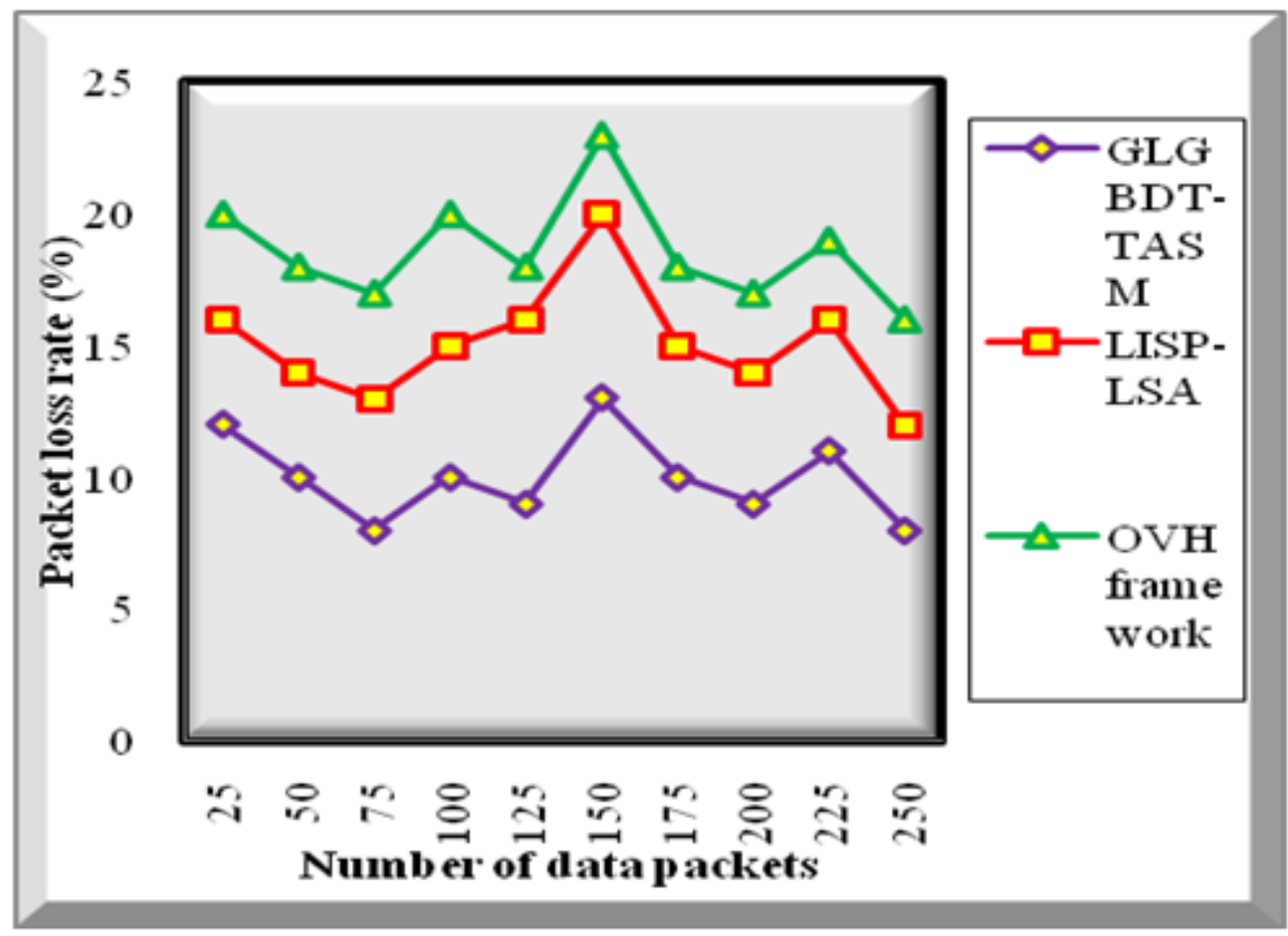

Figure 5 Simulation results of packet loss rate

Figure 5 shows the simulation results of the packet loss rate versus a number of data packets. For each run, the different results of the data packet loss rate are obtained as shown in figure 5. The graphical representation shows a comparatively lesser packet loss rate using the proposed GLGBDT-TASM model. This is because of measuring the bandwidth availability between the nodes. The higher bandwidth utilization of the nodes leads to cause more traffic while transmitting the data packets. Due to the network traffic, some of the data packets are lost. This helps to minimize successful data delivery. Therefore, the GLGBDT-TASM model finds the maximum bandwidth availability based on the total bandwidth and consumed bandwidth for efficient data transmission between the nodes. This helps to improve the seamless data transmission and minimize data loss.

Let consider ' 25 ' data packets for simulation, the GLGBDT-TASM model lost 3 data packets whereas the 4 and 5 data packets are lost using LISP-LSA [1] and OVH framework [2]. The loss rate of the proposed model is $12 \%$ and the loss rates of other methods are $16 \%$ and $20 \%$ respectively. Hence, the data packet loss is found to be lesser using GLGBDT-TASM model by $34 \%$ when compared to [1] and $46 \%$ when compared to [2].

\subsection{Impact of Data transmission delay}

Transmission delay is the time difference between the mobile nodes transmits and receives the data packets from the mobile node. The mathematical formula for data transmission delay is mathematically computed as follows,

$$
D T D=\left(T_{R}-T_{T}\right)(12)
$$

From (12),DTD denotes a data transmission delay, $T_{R}$ denotes data packets receiving the time, $T_{R}$ denotes a data packet sending time. The data transmission delay is measured in the unit of milliseconds (ms).

\section{Sample calculation}

Proposed GLGBDT-TASM: Let us consider a number of the data packet sent is 25, time for receiving the data packet is $14 \mathrm{~ms}$ and a time for sending the data packet from the node is $0 \mathrm{~ms}$. Then the data transmission delay is calculated as follows,

$$
D T D=14 m s-0 m s=14 m s
$$

Existing LISP-LSA scheme: Let us consider a number of the data packet sent is 25, time for receiving the data packet is $16 \mathrm{~ms}$ and a time for sending the data packet from the node is $0 \mathrm{~ms}$. Then the data transmission delay is calculated as follows,

$$
D T D=16 m s-0 m s=16 m s
$$

Existing OVH framework: Let us consider a number of the data packet sent is 25 , time for receiving the data packet is $19 \mathrm{~ms}$ and a time for sending the data packet from the node is $0 \mathrm{~ms}$. Then the data transmission delay is calculated as follows,

$$
D T D=19 m s-0 m s=19 m s
$$




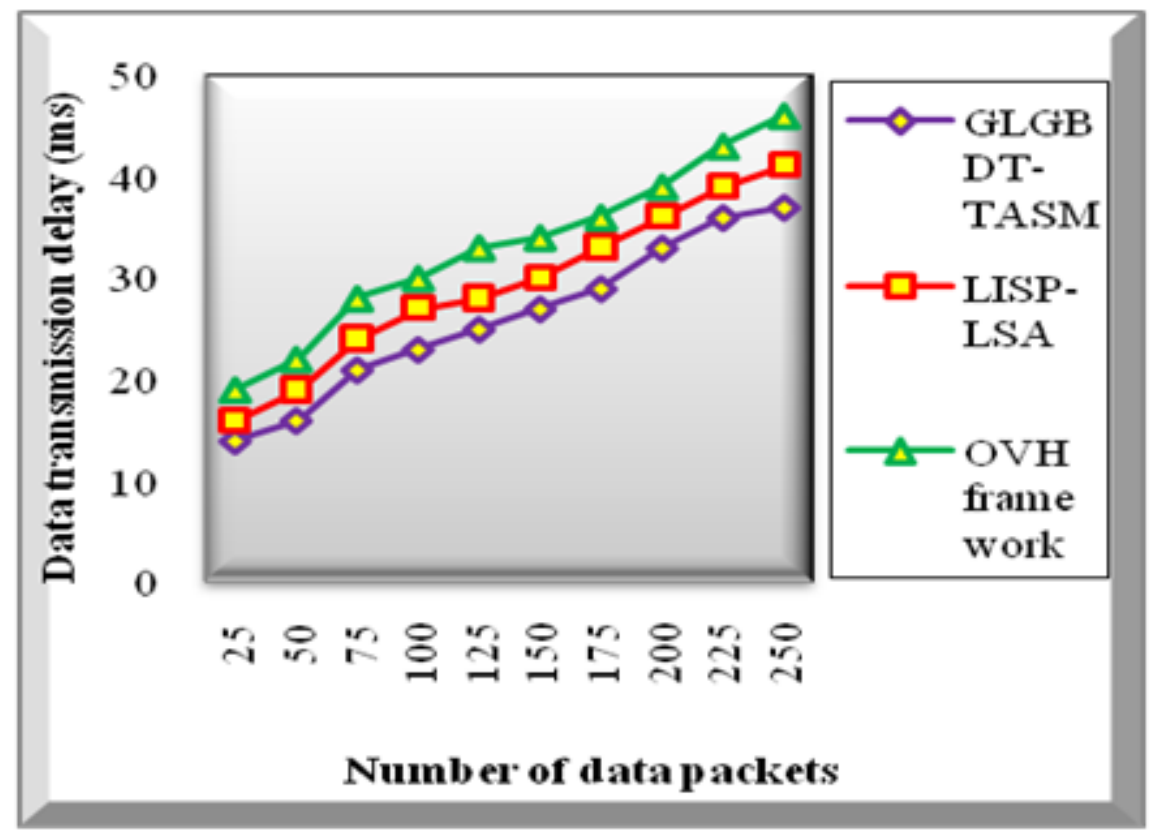

Figure 6 Simulation results of data transmission delay

Figure 6 illustrates the graphical representation of data transmission delay using three different methods namely GLGBDT-TASM, LISP-LSA [1] and OVH framework [2]. From the above information, it is concluded that the data transmission delay was found to be comparatively lesser using the GLGBDT-TASM model when compared to existing results. This is due to the process of handover and traffic-aware data transmission. By applying these two processes, not only, improves the data transmission and packet loss and also minimizing the delay. The selection of nearby available attachment points is selected for switching the node to perform seamless data delivery resulting in minimizing the transmission delay. In addition, the available bandwidth between the nodes is also taken into consideration for efficient data transmission. With this, the data transmission delay is reduced

For example, 25 data packets are considered for calculating the data transmission delay. The GLGBDTTASM model receives the data packets with $14 \mathrm{~ms}$ of delay and the other method receives the packets with $16 \mathrm{~ms}$ and $20 \mathrm{~ms}$ of delay. Similarly, the nine remaining runs are carried out with a different number of data packets. The results of data transmission delay using the GLGBDT-TASM model is compared to the existing results. The comparison results prove that the data transmission delay using the GLGBDT-TASM model is minimized by $11 \%$ and $22 \%$ when compared to LISP-LSA [1] and OVH framework [2] respectively.

The above discussion of various parameter results evidently shows that the GLGBDT-TASM model effectively performs the traffic-aware seamless mobility management in heterogamous networks with higher delivery and minimum delay as well as packet loss rate.

\section{CONCLUSION}

An efficient model called GLGBDT-TASM is introduced to minimize the traffic control loss and to handle seamless mobility in a heterogeneous network with lesser transmission delay. The ensemble classification technique determines the signal strength of the mobile nodes by constructing the base classifier with a minimum error rate. After the classification, seamless and continuous communication between the mobile nodes has to be performed through the handover from one point to another that improves the data delivery. In addition, the available bandwidths between the nodes are calculated to minimize the network traffic as well as reduce the packet loss. Simulation is carried out with different parameters such as seamless data delivery rate, packet loss rate, and transmission delay. The simulation results confirm that the GLGBDT-TASM model improves seamless mobility management in the heterogeneous network and minimizes the delay as well as the packet loss rate as compared to state-of-the-art methods. 


\section{References}

[1] Eric Mayeul Olusegun Fafolahan and Samuel Pierre, “A Seamless Mobility Management Protocol in 5G Locator Identificator Split Dense Small Cells”, IEEE Transactions on Mobile Computing, Pages 1-14, 2019.

[2] Xiaohong Li, Feng Liu, Zhiyong Feng, Guangquan Xu, Zhangjie Fu, “A novel optimized vertical handover framework for seamless networking integration in cyber-enabled systems”, Future Generation Computer Systems, Elsevier, Volume 79, Part 1, 2018, Pages 417-430, 2018

[3] Bingtao Yang, Xue Wang, Zhihong Qian, “A Multi-Armed Bandit Model-Based Vertical Handoff Algorithm for Heterogeneous Wireless Networks”, IEEE Communications Letters, Volume 22, Issue 10, Pages 2116 - 2119, 2018.

[4] Hassan Sinky, Bechir Hamdaoui, and Mohsen Guizani,” Proactive Multi-Path TCP for Seamless Handoff in Heterogeneous Wireless Access Networks”, IEEE Transactions on Wireless Communications, Volume 15, Issue 7, Pages 4754 - 4764, July 2016.

[5] Maram Alajlan, Abdelfettah Belghith, "Supporting Seamless Mobility for Real-Time Applications in Named Data Networking" Procedia Computer Science, Elsevier, Volume 110, Pages 62-69, 2017.

[6] T.Velmurugan, Sibaram Khara, S.Nandakumar, B.Saravanan, "Seamless Vertical Handoff using Invasive Weed Optimization (IWO) algorithm for heterogeneous wireless networks”, Ain Shams Engineering Journal, Elsevier, Volume 7, Issue 1, Pages 101-111, 2016.

[7] Khalid M. HosnyID, Marwa M. Khashaba, Walid I. KhedrID, Fathy A. Amer, "New vertical handover prediction schemes for LTEWLAN heterogeneous networks”, PLoS ONE, Volume 14, Issue 4, Pages 1-31, 2019.

[8] Cheng-Wei Lee, Meng Chang Chen, Yeali S.Sun, "A novel network mobility management scheme supporting seamless handover for high-speed trains”, Computer Communications, Elsevier, Volume 37, Pages 53-63, 2014.

[9] Ákos Leiter and László Bokor, "A Flow-Based and Operator-Centric Dynamic Mobility Management Scheme for Proxy Mobile IPv6”, Wireless Communications and Mobile Computing, Hindawi, Volume 2019, Pages 1-2, April 2019.

[10] Shilpa R.Litake and Dr. Prachi Mukherji, "Modeling Seamless Vertical Handovers in Heterogeneous Wireless Networks", International Journal on Future Revolution in Computer Science \& Communication Engineering, Volume 3 Issue 11, Pages 524-53, 2017.

[11] B.R.Chandavarkar and Ram Mohana Reddy Guddeti, "Simplified and improved multiple attributes alternate ranking method for vertical handover decision in heterogeneous wireless networks”, Computer Communications, Elsevier, Volume 83, Pages 81-97, 2016.

[12] Khaled Zeraoulia and Nadjib Badache, "SM-SIP: Seamless Mobility Management for Heterogeneous Wireless Networks", International Journal of Computer Network and Information Security, Volume 9, Pages 58-65, 2014.

[13] Ali Safa Sadiq, Norsheila Binti Fisal, Kayhan Zrar Ghafoor, and Jaime Lloret, "An Adaptive Handover Prediction Scheme for Seamless Mobility Based Wireless Networks”, The Scientific World Journal, Hindawi Publishing Corporation, Volume 2014, Pages 117, December 2014.

[14] Nouri Omheni, Imen Bouabidi, Amina Gharsallah, Faouzi Zarai, Mohammad S. Obaidat, "Smart mobility management in 5G heterogeneous networks”, IET Networks, Volume 7, Issue 3, Pages 119 - 128, 2018.

[15] Alex Monteiro, Eduardo Souto, Richard Pazzi, Michele Nogueira, "Context-aware network selection in heterogeneous wireless networks”, Computer Communications, Elsevier, Volume 135, Pages 1-15, 2019.

[16] Jian Zhou and Can-yan Zhu, "Compensatory Analysis and Optimization for MADM for Heterogeneous Wireless Network Selection", Journal of Electrical and Computer Engineering, Hindawi Publishing Corporation, Volume 2016, Pages 1-9, May 2016.

[17] Md. Jalil Piran, Nguyen H. Tran, Doug Young Suh, Ju Bin Song, Choong Seon Hong, Zhu Han, "QoE-Driven Channel Allocation and Handoff Management for Seamless Multimedia in Cognitive 5G Cellular Networks”, IEEE Transactions on Vehicular Technology, Volume 66, Issue 7, Pages 6569 - 6585, 2017.

[18] Ravikumar Balakrishnan and Ian Akyildiz, "Local Anchor Schemes for Seamless and Low-cost Handover in Coordinated Small Cells”, IEEE Transactions on Mobile Computing, Volume 15, Issue 5, Pages 1182 - 1196, 2016

[19] Jozef Wozniak, "Mobility management solutions for current IP and future networks", Telecommunication Systems, Springer, Volume 61, Issue 2, Pages 257-275, 2016.

[20] Aabha Jaina and SanjivTokekar Dr, “Application Based Vertical Handoff Decision in Heterogeneous Network”, Procedia Computer Science, Elsevier, Volume 57, Pages 782-788, 2015.

[21] Dhanaraj Cheelu, Rajasekhara Babu Madda, Chi-Yuan Chen, P. Venkata Krishna, Sumanth Yenduri, “ Intelligent vertical handoff decision strategy based on network performance prediction and consumer surplus value for next generation wireless networks", IET Networks, Volume: 6 , Issue: 4 , 2017.

[22] Wahida Mansouri, Faouzi Zarai, Kais Mnif, Lotfi Kamoun, "Cross layer architecture with integrated MIH in heterogeneous wireless networks” Computer Networks, Volume 127, Issue:9, Pages 126-137, November 2017.

[23] Vasudeva, K., Simsek, M., Lopez-Perez, D., \& Guvenc, I, “Analysis of Handover Failures in Heterogeneous Networks With Fading”, IEEE Transactions on Vehicular Technology, Volume 66, Issues 7, Pages 1-14, 2017.

[24] Shi, F., Li, K., \& Shen, Y, “ Seamless handoff scheme in Wi-Fi and WiMAX heterogeneous networks", Future Generation Computer Systems”, Volume 26, Issues 8, Pages 1403-1408, 2010. 